\title{
On the Performance of Delay-Tolerant Routing Protocols in Intermittently Connected Mobile Networks
}

\author{
Md. Sharif Hossen* and Muhammad Sajjadur Rahim \\ Department of Information and Communication Engineering, University of Rajshahi, \\ Rajshahi 6205, Bangladesh.
}

*Corresponding Author: sharif5613@gmail.com

\begin{abstract}
Delay-Tolerant Networks are used to enable communication in challenging environments where nodes are intermittently connected, and an end-to-end path does not exist all the time between source and destination, e.g., Intermittently Connected Mobile Networks (ICMNs). Therefore, network environments, where the nodes are characterized by opportunistic connectivity, are appropriately modeled as Delay-Tolerant Networks (DTNs). In this paper, we have investigated the performance of DTN routing protocols, namely Epidemic, PRoPHET, and Spray-and-Wait (Binary version) in an ICMN scenario. Their performances are analyzed in terms of delivery probability, average latency, and overhead ratio of varying message generation rates and number of mobile nodes, respectively. In addition, the impacts of varying buffer size and Time-to-Live (TTL) on their performances are investigated. For evaluating these performance metrics, we have used Opportunistic Network Environment (ONE) simulator as the simulation tool. The outcome of this work shows that for the ICMN scenario, the best DTN routing technique is Binary Spray-and-Wait, whereas Epidemic routing exhibits the worst performance in terms of all the metrics considered here.
\end{abstract}

Keywords: Delay-tolerant network (DTN); intermittently connected mobile network (ICMN); opportunistic network environment (ONE) simulator; epidemic; PRoPHET; binary spray-and-wait; routing; simulation

\section{INTRODUCTION AND RELATED WORKS}

Transfer of data from source to destination is the fundamental ability that all communication networks must adhere to. Delay- and Disruption-Tolerant Networks, generally known as DTNs, are characterized by their lack of connectivity due to node mobility and typically sparse topologies, network partitions, long and variable propagation delay, and high bit error rate of the communication channels [1]. These circumstances eventually lead to the lack of an instantaneous end-to-end path from source to destination. In such challenging environments, popular ad-hoc routing protocols such as Ad hoc On-Demand Distance Vector (AODV) [2] and Dynamic Source Routing (DSR) [3] cannot be implemented for routing data successfully, as a continuous end-toend path is needed before forwarding data packets through the network.

Intermittently Connected Mobile Networks (ICMNs) [4], also referred to as DTNs, feature intermittent connectivity and temporarily broken links, which prevent the common ad-hoc routing protocols to transfer data from source to destination successfully [5]. When instantaneous end-to-end paths do not exist, the routing protocols must adopt the "store-carry-and-forward" approach, which exploits the mobility of nodes to route data. In this approach, data is moved from the source to the next available node and stored there, waiting for an opportunity to forward the data. If present, mobile nodes can carry the 
stored data while moving, and search for opportunities to forward the data to other nodes towards the destination. Overall, these techniques provide eventual data delivery with a certain probability [6]-[8]. The delivery probability of the packets can be maximized by replicating them to different nodes so that at least one of the copies will successfully reach its destination with high probability [9]. Routing protocols that behave this way are called replication-based. And protocols that do not replicate packets are called forwarding-based [10].

In this paper, we present an evaluation of DTN routing protocols as applied to intermittently connected mobile networks, with focus on Epidemic [9], PRoPHET [11], and Spray-and-Wait [12]; which is an enhanced version of our previous research work [13]. Epidemic routing is an obvious example of the "store-carry-and-forward" approach, which forwards each copy of the messages (not common among the nodes in contact) to each node. This approach is flooding-based in nature, and is exhaustive of network resources. The Probabilistic Routing Protocol using History of Encounters and Transitivity (PRoPHET) attempts to exploit the likelihood of real-world encounters by maintaining a set of probabilities for successful delivery to known destinations in the DTN, and replicates messages during opportunistic encounters only if the mobile node in contact that does not possess the message appears to have a better chance of delivering it to the destination. Spray-and-Wait approach is proposed by Spryropoulos et al. in [12] to limit the utilization of network resources, in which only a limited number of message copies are replicated among nodes. It parts routing into two phases: a spray phase, where message replicas are spread, and a wait phase, where nodes with single-copy messages wait until a direct encounter with the respective destinations. The rest of this paper is organized as follows. Next we describe the DTN routing protocols investigated in this paper followed by the description of ONE simulator and simulation setup. Then we explain the analysis of the obtained results, and finally we provide the concluding remarks about this research endeavor.

\section{Description of the Routing Protocols under InVEStigation}

In this section, we give a brief overview on the classification of DTN routing protocols, and summarize the design of the Epidemic, PRoPHET, and Spray-and-Wait routing protocols.

DTN routing protocols are categorized into two basic schemes: single-copy and multicopy. In single-copy schemes, a single copy of each message is forwarded through the network, which is called forwarding-based routing. Multi-copy schemes forward several copies of the same message to the network, i.e., replicate messages, and hence are called replication-based. There are merits and demerits to both the schemes, and which scheme is preferable depends on the application scenario. Forwarding-based routing scheme is generally resource efficient as only a single copy of a message exists in the network at any given time, but it does not guarantee the best delivery ratio since the probability of finding the destination node is low and usually exhibits high latency [10]. On the other hand, replication-based routing protocols obtain higher message delivery ratios [6], since several copies of messages exist in the network, and at least one must reach the destination. Therefore, there is a typical tradeoff found between the two schemes, whereby the former spends less resources but may provide low probability of successful delivery, whereas the latter tends to spend more resources but also provides better delivery ratios [14]. 
Epidemic routing widens the idea of flooding in intermittently connected mobile networks. It is one of the first algorithms proposed to enable message delivery in such networks. Epidemic routing is flooding based where every node continuously replicates a copy of message to all nodes it encounters that do not have in common the message copy. So this algorithm is the best-effort approach to reach the destination compared to flooding which forwards a copy of every data packet to every node [12].

The basic operation of PRoPHET routing is similar to Epidemic and attempts to improve the delivery probability of messages. Message forwarding is based on the calculation of probability (also called delivery predictability) by each node to each destination node. When two nodes are encountered, messages are forwarded to a node that has higher delivery predictability. Delivery predictability $P_{(a, b)}$ is stored in internal delivery vector and gets updated whenever nodes meet each other. The delivery predictabilities used by each node are recalculated at each opportunistic encounter according to three rules:

(i) When node $\mathrm{A}$ encounters another node $\mathrm{B}$, the predictability for $\mathrm{B}$ is increased. Equation (1) shows this calculation.

$$
P_{(a, b)}=P_{(a, b) \text { old }}+\left(1-P_{(a, b) \text { old }}\right) \times P_{\text {init }}
$$

where $P_{\text {init }}$ is an initialization constant.

(ii) The delivery predictability must age because if two nodes do not encounter each other in a while, then they are less likely to forward messages to each other. Equation (2) shows this ageing equation.

$$
P_{(a, b)}=P_{(a, b) o l d} \times \gamma^{k}
$$

where $\gamma^{k}$ is an aging constant.

(iii) The delivery predictability also follows the transitive property, that is, if a node A frequently encounters node B and node B frequently encounters node D, then node D probably is a good node to forward message intended for node A. Equation (3) shows the effect of transitivity on delivery predictability.

$$
P_{(a, d)}=P_{(a, d) \text { old }}+\left(1-P_{(a, d) \text { old }}\right) \times P_{(\mathrm{a}, \mathrm{b})} \times P_{(b, d)} \times \beta(3)
$$

where $\beta$ is a scaling constant that decides how large impact the transitivity should have on the delivery predictability.

Spray-and-Wait (SNW) routing protocol is a class of replication-based schemes that attempts to find a good delivery ratio by limiting the number of replicas of a given message while keeping resource utilization low as in forwarding-based routing. SNW achieves resource efficiency by setting a fixed upper bound on the number of copies per message allowed in the network. The SNW protocol consists of the following two phases:

- Spray Phase: For every message originating at a source node, L message copies are initially spread - forwarded by the source and possibly other nodes receiving a copy - to L distinct "relays".

- Wait Phase: If the destination is not found in the spraying phase, each of the L nodes carrying a message copy performs direct transmission (i.e., will forward the message only to its destination).

There are two main versions of the SNW routing protocol, respectively known as Vanilla and Binary. The two versions differ in the mechanism employed to "spray" the L copies of a message. To achieve this, a simplest way called Vanilla is to transmit a single copy of the message from the source to the first L distinct nodes it encounters after the message is generated. The second version, referred to as Binary, works as follows: the source node 
starts with L copies of the message. It transfers L/2 of the copies to the first node it encounters. Each node then transfers half of its copies to future nodes they meet that have no copy of the message. When a node eventually gives away all of its copies, except for one, it switches to the wait phase where it waits for a direct transmission opportunity with the final destination of the message. The advantage of the Binary version is that messages are disseminated much faster than in the Vanilla version.

\section{SIMULATION TOOL AND SETUP}

In this paper, we focus on the performance analysis of Epidemic, PRoPHET, and Sprayand-Wait (Binary version) routing protocols in an intermittently connected mobile network. All routing protocols are simulated using Opportunistic Network Environment (ONE) [15] with program version of 1.5.1. This section explains ONE simulator as the simulation tool and the network environment modeling parameters for simulation setup.

\section{$A$. The ONE Simulator}

ONE is an agent-based discrete event simulation engine that is designed for evaluating the performance of DTN routing protocols. Unlike other DTN simulators, which usually focus only on routing simulation, the ONE combines mobility modeling, inter-node contacts, DTN routing, message handling and visualization in one package that is easily pluggable, extendable and runnable on different platforms with no or little modification and provides a rich set of reporting and analyzing modules. A detailed description of the simulator is available in [15] and the ONE simulator project page [16] where the source code of the simulator is also available. Source codes are written in Java programming language.

\section{B. Simulation Setup}

We consider the following parameters of simulation setup and routing algorithms. Table I shows the simulation configuration for analyzing the message generation rate and number of nodes, respectively. For varying message generation rate, the total number of nodes is 126, where 80 pedestrians (two groups with 40 nodes in each group), 40 cars (single group of 40 nodes), and 6 trams (three groups with 2 nodes in each group). Again, for varying the number of nodes, message generation rate is 2 , i.e., one new message in every 25 to 35 seconds or two messages per minute.

TABLE I. SIMULATION PARAMETERS FOR VARYING MESSAGE GENERATION RATES AND NUMBER OF MOBILE NODES

\begin{tabular}{|c|c|}
\hline Parameters & Values \\
\hline Simulation Time & 24 hours \\
\hline Update Interval & 1 second \\
\hline Number of Nodes in Group & $50,60,80,100,150,300,400$ \\
\hline Interface & Bluetooth Interface \\
\hline Interface Type & Simple Broadcast Interface \\
\hline Transmit Speed & $250 \mathrm{kbps}$ \\
\hline Transmit Range & $10 \mathrm{~m}$ \\
\hline Routing Protocols & Epidemic, PRoPHET, and B-SNW \\
\hline
\end{tabular}


On the Performance of Delay-Tolerant Routing Protocols in Intermittently Connected Mobile

\begin{tabular}{|l|l|}
\hline \multicolumn{1}{|c|}{ Parameters } & \multicolumn{1}{c|}{ Values } \\
\hline Buffer Size & $5 \mathrm{MB}$ \\
\hline Message Generation Rate (message/min.) & $1.5,2,2.5,3,4,5,6,7.5,10$ \\
\hline Message TTL & 300 minutes \\
\hline Mobility & Random Way Point \\
\hline Movement model & Shortest Path Map Based \\
\hline Message Size & $500 \mathrm{~KB}-1 \mathrm{MB}$ \\
\hline Simulation Area Size & $4500 \mathrm{~m} \times 3400 \mathrm{~m}$ \\
\hline
\end{tabular}

Table II shows the simulation configuration for analyzing the buffer size and Time-to-Live (TTL), respectively. For varying buffer size (i.e. 5MB, 10MB, 15MB, 20MB, and $25 \mathrm{MB}$ ), we have considered TTL constant with 300 minutes. Again, for varying TTL (i.e. 50, 100, 150, 200, 250, and 300 minutes), buffer size is $30 \mathrm{MB}$. Table III summarizes the simulation configuration for routing algorithms.

\begin{tabular}{|c|c|c|}
\hline \multirow{2}{*}{\multicolumn{3}{|c|}{\begin{tabular}{c|c} 
TABLE II. SIMULATION PARAMETERS FOR VARYING \\
Parameters & Values
\end{tabular}}} \\
\hline & & \\
\hline Simulation Time & \multicolumn{2}{|l|}{24 hours } \\
\hline Update Interval & \multicolumn{2}{|l|}{1 second } \\
\hline Number of Nodes & \multicolumn{2}{|l|}{126} \\
\hline Interface & \multicolumn{2}{|l|}{ Bluetooth Interface } \\
\hline Interface Type & \multicolumn{2}{|c|}{ Simple Broadcast Interface } \\
\hline Transmit Speed & \multicolumn{2}{|l|}{$250 \mathrm{kbps}$} \\
\hline Transmit Range & \multicolumn{2}{|l|}{$10 \mathrm{~m}$} \\
\hline Routing Protocols & \multicolumn{2}{|c|}{ Epidemic, PRoPHET, B-SNW } \\
\hline Buffer Size & \multicolumn{2}{|c|}{$5 \mathrm{MB}, 10 \mathrm{MB}, 15 \mathrm{MB}, 20 \mathrm{MB}, 25 \mathrm{MB}$} \\
\hline Message Generation Rate & \multicolumn{2}{|c|}{2 , i.e., one message in $25-35$ seconds } \\
\hline Message TTL & \multicolumn{2}{|c|}{$50,100,150,200,250,300$ (minutes) } \\
\hline Mobility & \multicolumn{2}{|l|}{ Random Way Point } \\
\hline Movement model & \multicolumn{2}{|l|}{ Shortest Path Map Based } \\
\hline Message Size & \multicolumn{2}{|l|}{$500 \mathrm{~KB}-1 \mathrm{MB}$} \\
\hline Simulation Area Size & \multicolumn{2}{|l|}{$4500 \mathrm{~m} \times 3400 \mathrm{~m}$} \\
\hline TABLE III. & \multicolumn{2}{|c|}{ ARAMETERS OF ROUTING ALGORITHMS } \\
\hline Routing Algorithms & Parameters & Values \\
\hline Epidemic & N/A & N/A \\
\hline PRoPHET & Seconds in Time Unit & $30 \mathrm{~s}$ \\
\hline Binary Spray-and-Wait & No. of Copies (L) & 6 \\
\hline
\end{tabular}




\section{RESULTS AND ANALYSIS}

We have mainly analyzed three performance metrics of DTN routing protocols: delivery probability, average latency, and overhead ratio for four cases as follows:

(i) for varying message generation rate with constant number of mobile nodes, (ii) for varying number of mobile nodes with constant message generation rate, (iii) for varying buffer size with constant TTL, and (iv) for varying time-to-live (TTL) with constant buffer size. Time-to-Live (TTL) is a mechanism that limits the lifetime of a data packet in a network. The packet will keep on being copied from one node to the other node until its TTL expires.

\section{A. Delivery Probability}

Delivery probability is the ratio of the total number of messages delivered to the destination over the total number of messages created at the source.

$$
\text { Delivery probability }=\frac{\text { Number of messages delivered }}{\text { Number of messages created }}
$$

From Fig. 1, we see that Binary Spray-and-Wait (B-SNW) protocol achieves better performance than Epidemic and PRoPHET in terms of delivery probability for every message generation rates. From Fig. 2, it is evident that the delivery probability of BSNW is much better than both Epidemic and PRoPHET routing protocols for each setting of node groups. In both the cases, i.e., varying message generation rate and number of nodes per group, the lowest delivery ratio is provided by Epidemic routing protocol as it does not apply any strategy for replicating messages.

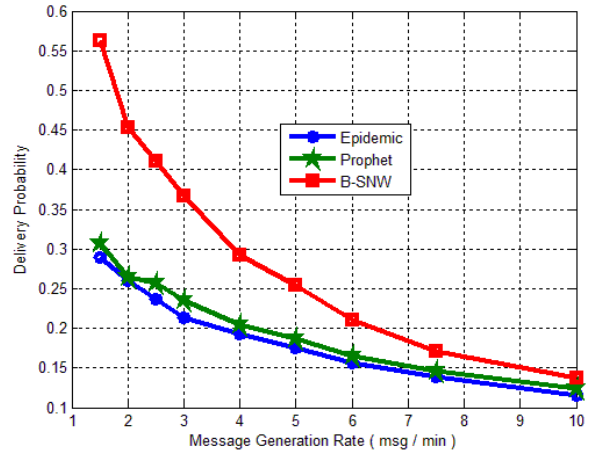

Fig. 1. Delivery probability vs. message generation rate

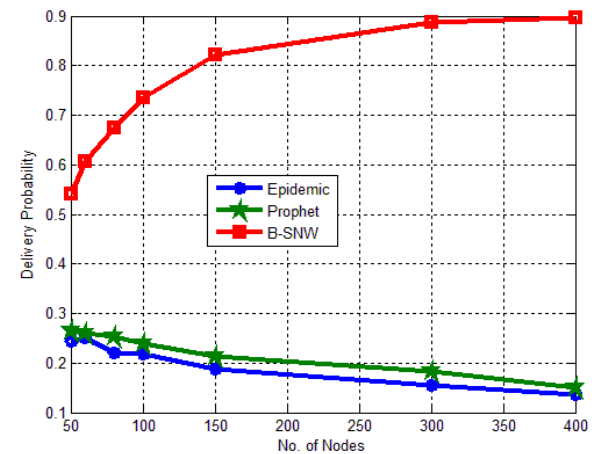

Fig. 2. Delivery probability vs. number of nodes per group

With increasing buffer size, more message copies can be stored within a node, and hence delivery probability increases. From Fig. 3, we see that B-SNW protocol achieves better performance than Epidemic and PRoPHET in terms of delivery probability for varying buffer sizes because it occupies less wasteful message copies. From Fig. 4, it observed that B-SNW gives the better results as compared to Epidemic and PRoPHET for varying TTL. In both cases, i.e., varying buffer sizes and TTL, the B-SNW achieves highest delivery ratio but Epidemic routing shows the lowest delivery as it does not apply any strategy for replicating messages. 


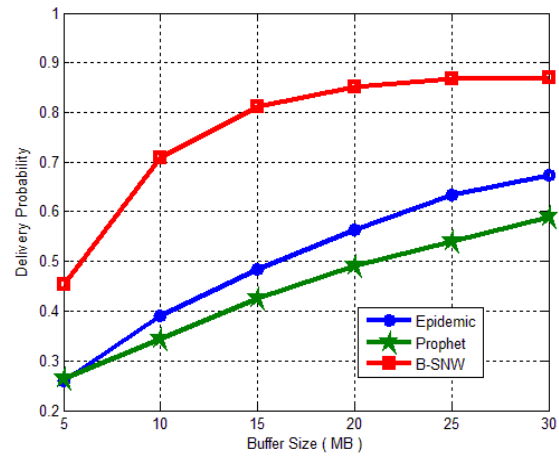

Fig. 3. Delivery probability vs. buffer size

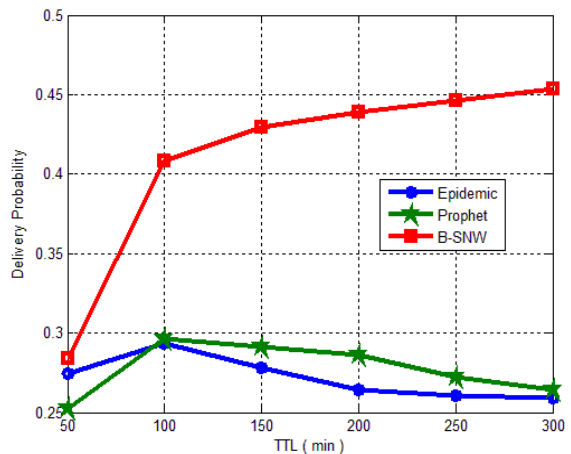

Fig. 4. Delivery probability vs. TTL

\section{B. Average Latency}

Average latency is the measure of average time between messages generated and messages received by destination node.

$$
\text { Average latency }=\quad \sum_{i=1}^{n} \frac{\text { Time when message received }- \text { Time when message produced }}{\text { Number of messages received }}
$$

From Fig. 5, we see that average latency decreases in accordance with the increase in message generation rates for all routing protocols and B-SNW has higher performance than others do. While as the number of nodes increases as shown in Fig. 6, the average latency of Epidemic and PRoPHET routing decreases but increases in case of B-SNW. From Fig. 7, we see that average latency increases gradually in accordance with the increase in buffer sizes for all routing protocols. However, we see that B-SNW has the lowest average latency and thus it shows better performance than Epidemic and PRoPHET. As shown in Fig. 8, average latency increases with the increase of TTL for all routing protocols. Here, B-SNW obtains better performance in both the cases.

\section{Overhead Ratio}

The overhead ratio defines how many redundant packets are relayed to deliver one packet. It simply reflects the cost of transmission in a network, and is defined as follows:

$$
\text { Overhead ratio }=\frac{\mathrm{R}-\mathrm{D}}{\mathrm{D}}
$$

where $\mathrm{R}$ is the number of messages forwarded by relay nodes, and $\mathrm{D}$ is the number of messages delivered to their destination. 


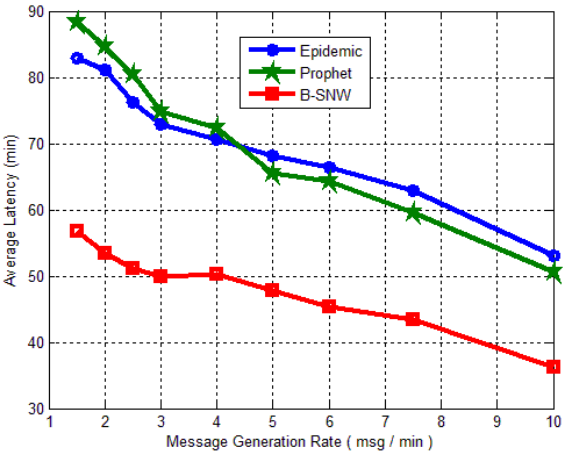

Fig. 5. Average latency vs. message generation rate

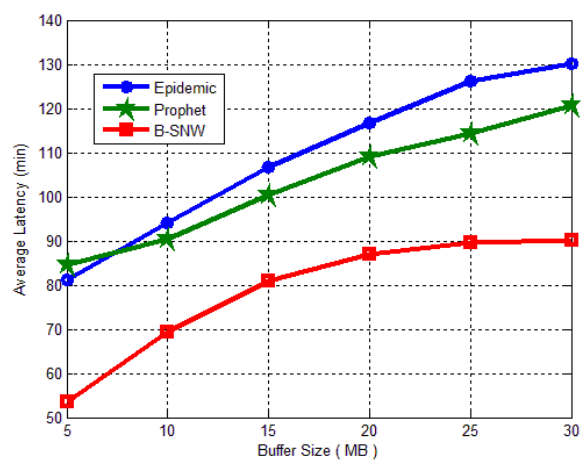

Fig. 7. Average latency vs. buffer size

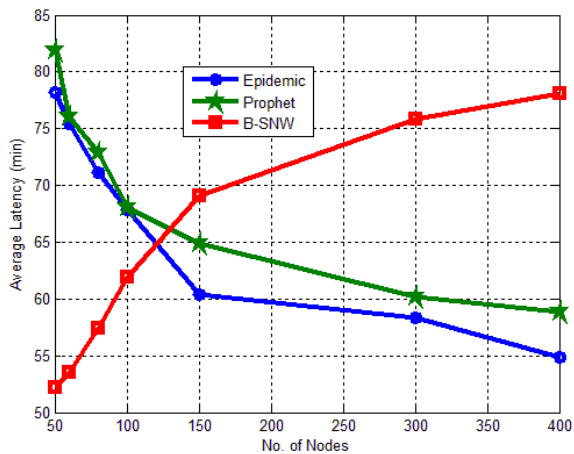

Fig. 6. Average latency vs. number of nodes per group

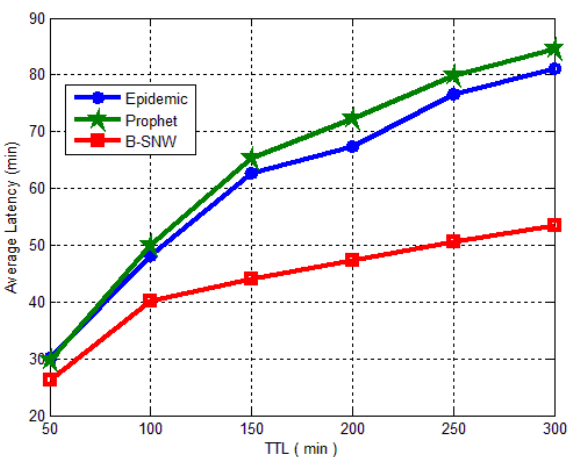

Fig. 8. Average latency vs. TTL

From Figs. 9 and 10, we see that Epidemic routing exhibits the highest value of overhead ratio compared to others due to its resource-consuming message forwarding mechanism, whereas B-SNW has the lowest overhead ratio due to limiting the number of message copies during spray phase. From Figs. 11 and 12, we observe that Epidemic routing exhibits the highest value of overhead ratio compared to others due to its resourceconsuming message forwarding mechanism. However, in both cases as shown in Figs. 11 and 12 , B-SNW has the lowest overhead ratio. Hence, in case of overhead ratio, B-SNW also shows the better performance than other DTN routing protocols.

\section{CONCLUSIONS}

In this paper, we have investigated the performance of DTN routing protocols, i.e., Epidemic, PRoPHET, and Spray-and-Wait (Binary version), in intermittently connected mobile networks (ICMNs). Simulation results show the performance comparison of the investigated DTN routing protocols in terms of message delivery probability, average latency and overhead ratio with the variation of message generation rates, number of 


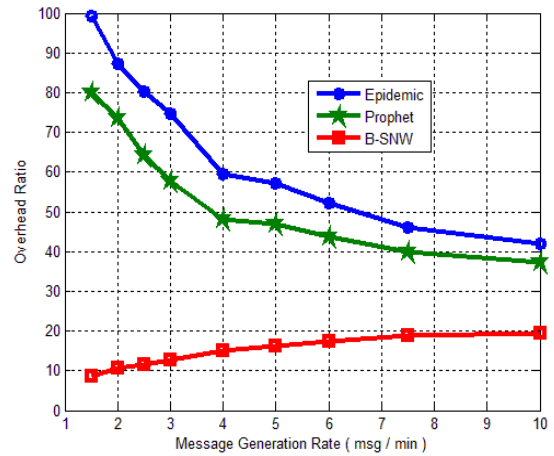

Fig. 9. Overhead ratio vs. message generation rate

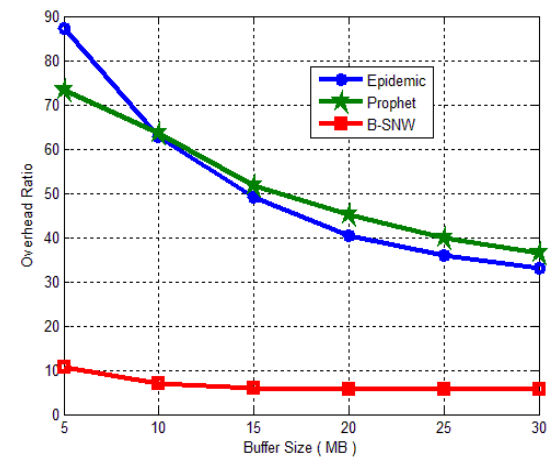

Fig. 11. Overhead ratio vs. buffer size

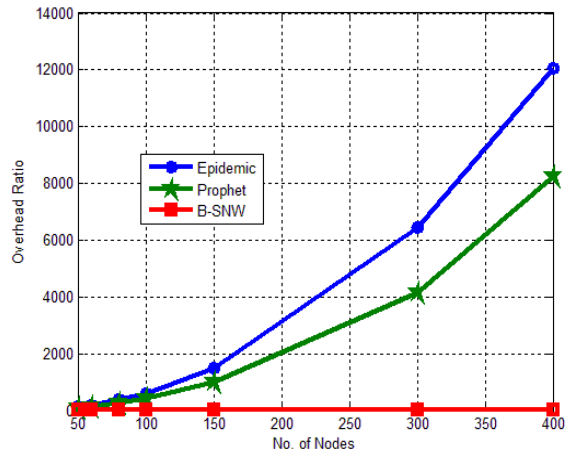

Fig. 10. Overhead ratio vs. number of nodes per group

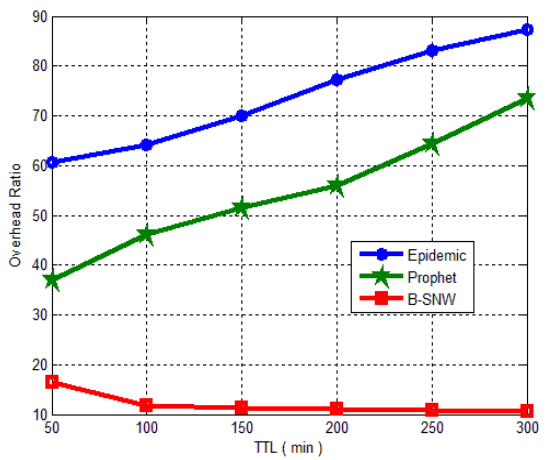

Fig. 12. Overhead ratio vs. TTL

nodes, buffer size and TTL, respectively. From these results, we may conclude that the best candidate for routing messages in ICMNs is Binary Spray-and-Wait.

\section{REFERENCES}

[1] K. Fall, "A delay-tolerant network architecture for challenged internets," in Proc. of ACM SIGCOMM, Karlsruhe, Germany, Aug. 2003, pp. 27-34.

[2] C. E. Perkins and E. M. Royer, "Ad hoc on-demand distance vector routing," in Proc. of the 2nd IEEE Workshop on Mobile Computing Systems and Applications, New Orleans, LA, Feb. 1999, pp. 90-100.

[3] D. B. Johnson and D. A. Maltz, "Dynamic source routing in ad hoc wireless networks," Mobile Computing, Kluwer Academic Publishers, Feb. 1996, ch.5, pp. 153-181.

[4] L. Pelusi, A. Passarella, and M. Conti, "Opportunistic networking: data forwarding in disconnected mobile ad hoc networks," IEEE Comm. Mag., vol. 44, no. 11, pp. 134-141, Nov. 2006.

[5] Z. Zhang, "Routing in intermittently connected mobile ad hoc networks and delay tolerant networks: overview and challenges," in Proc. of IEEE Communication Surveys and Tutorials, vol. 8, no. 1, pp. 24-37, Jan. 2006. 
[6] J. Burgess, B. Gallagher, D. Jensen, and B. N. Levine, "MaxProp: routing for vehicle-based disruption-tolerant networks," in Proc. of IEEE INFOCOM, Barcelona, Spain, Apr. 2006.

[7] P. Juang, H. Oki, Y. Wang, M. Martonosi, L. S. Peh, and D. Rubenstein, "Energy-efficient computing for wildlife tracking: design tradeoffs and early experiences with zebranet," in Proc. of ASPLOS, San Jose, CA, 2002, pp. 96-107.

[8] A. Chaintreau, P. Hui, J. Crowcroft, C. Diot, R. Gass, and J. Scott, "Impact of human mobility on opportunistic forwarding algorithms," IEEE Transactions on Mobile Computing, vol. 6, no. 6, pp. 606-620, Jun. 2007.

[9] A. Vahdat and D. Becker, "Epidemic routing for partially connected ad hoc networks," Department of Computer Science, Duke University, Tech. Rep. CS-2000-06, Apr. 2000.

[10] S. Jain, K. Fall, and R. Patra, "Routing in a delay-tolerant network," in Proc. of ACM SIGCOMM, Portland, OR, 2004, pp. 145-157.

[11] A. Lindgren, A. Doria, and O. Scheln, "Probabilistic routing in intermittently connected networks," ACM Mobile Comput. And Commun. Review, vol. 7, no. 3, pp. 19-20, Jul. 2003.

[12] T. Spyropoulos, K. Psounis, and C. S. Raghavendra, "Spray and wait: an efficient routing scheme for intermittently connected mobile networks," in Proc. of ACM WDTN, Philadelphia, PA, Aug. 2005, pp. 252-259.

[13] M. S. Hossen and M. S. Rahim, "Performance analysis of delay-tolerant routing protocols in intermittently connected mobile networks," in Proc. of Int. Conf. on Matls., Elec. \& Info. Engg., Jun. 2015, Faculty of Engineering, University of Rajshahi, Rajshahi, Bangladesh.

[14] T. Spyropoulos, K. Psounis, and C. S. Raghavendra, "Spray and focus: efficient mobilityassisted routing for heterogeneous and correlated mobility," in Proc. of IEEE PerCom, White Plains, NY, Mar. 2007, pp. 79-85.

[15] A. Keränen, J. Ott, and T. Kärkkäinen, "The ONE simulator for DTN protocol evaluation," in Proc. of the 2nd Int. Conf. on Simul. Tools and Tech., 2009, Rome, Italy.

[16] Project page of the ONE simulator, [Online], https://www.netlab.tkk.fi/tutkimus/dtn/theone, (Accessed: 10 Nov., 2014). 